\section{Australian Journal of \\ Crop Science}

AJCS 15(03):334-342 (2021)

doi: 10.21475/ajcs.21.15.03.p2592
ISSN:1835-2707

\title{
Contribution of nitrogen on industrial quality of oat grain components and the dynamics of relations with yield
}

\begin{abstract}
Rubia Diana Mantai ${ }^{1 *}$, José Antonio Gonzalez da Silva ${ }^{2}$, Ivan Ricardo Carvalho ${ }^{2}$ Francine Lautenchleger ${ }^{3}$, Roberto Carbonera ${ }^{2}$, Luiz Antônio Rasia ${ }^{2}$, Adriana Roselia Kraisig ${ }^{2}$, Vanessa Pansera ${ }^{2}$, Odenis Alessi ${ }^{2}$, Juliana Aozane da Rosa ${ }^{2}$, Eduarda Warmbier², Natiane Carolina Ferrari Basso², Ester Mafalda Matter ${ }^{2}$
\end{abstract}

\author{
${ }^{1}$ Universidade Regional Integrada do Alto Uruguai e das Missões \\ ${ }^{2}$ Universidade Regional do Noroeste do Estado do Rio Grande do Sul \\ ${ }^{3}$ Universidade Estadual do Centro-Oeste
}

\section{*Corresponding author: rdmantai@yahoo.com.br}

\section{Abstract}

Understanding the magnitude of contribution and relationships of industrial quality components to yield by nitrogen stimulation can drive strategies with benefits to the food industry. The objective of this study is to measure and interpret the contribution and relationship dynamics of the components of oat industrial quality with grain and industry yield by nitrogen stimulation, partitioning the correlation values in direct and indirect effects by path diagnosis, in proposing strategies that promote benefits to the food industry. The study was conducted from 2011 to 2016, in a randomized block design with four replications in 4x2 factorial for nitrogen rates $\left(0,30,60\right.$ and $\left.120 \mathrm{~kg} \mathrm{ha}^{-1}\right)$ and oat cultivars (Barbarasul and Brisasul) in separate environments soybean/oat and corn/oat succession system. The increase of nitrogen promoted greater change in the mass of caryopsis in soybean/oat system and the thousand grain mass and number of grains greater than $2 \mathrm{~mm}$ in corn/oat system, with a tendency of reduction. In soybean/oat system, grain and industry yields can be simultaneously incremented by direct increase via one thousand grain mass and indirect increase by caryopsis mass. In corn/oat system the grain yield does not show any relationship with industrial quality variables. However, the industral productivity is benefited by the increase of the number of grains larger than $2 \mathrm{~mm}$. The management proposition in the improvement of the grain and industry productivity characteristics by nitrogen is dependent on the high succession and reduced $\mathrm{N}$-residual release systems.

Keywords: Avena sativa L., correlation and path, sustainability.

Abbreviations: $\mathrm{GY}_{\text {_ }}$ grain yield; IY _ industrial index; TGM _ thousand grain mass; $\mathrm{HM}_{\text {_ }}$ hectolitre mass; $\mathrm{NG}_{>2}$ _ number of grains

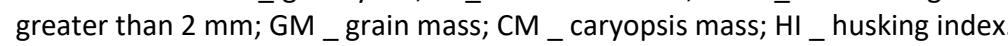

\section{Introduction}

White oat features prominent grains in human food, fostering a range of food processing industries (Martinez et al., 2010; Silveira et al., 2016). To obtain adequate grains to market demands, it is necessary from the production process to combine adequate genetics and management technologies to promote physical and nutritional modifications (Marolli et al., 2017; Aseeva and Melnichuk, 2018).

Among the management technologies, nitrogen fertilization is essential in the development and elaboration of oat grains, influencing components of productivity and industrial quality (Takayama et al., 2006; Obour et al., 2018). According to Tomple and Hwan (2018), the increase in grain production by the addition of nitrogen is due to the fact that nitrogen stimulates growth through high rates of photosynthesis. The main form of supply is via fertilization with urea in cover, on the surface and without incorporation, which, due to the high susceptibility to losses due to ammonia volatilization, can represent economic losses and environmental damage (Ziech et al., 2016).
The nutrient efficiency is related to cultivar genetics, supply dose, succession systems and growing weather conditions, changing the magnitude and expression of grain yield and explanatory variables of industrial yield. Grain yield, grain thickness greater than $2 \mathrm{~mm}$ and husking index (caryopsis mass over grain mass) are the main variables that define the industrial productivity of oat grains (Arenhardt et al., 2015; Scremin et al., 2017). White and Finnan (2017) highlight that higher doses of nitrogen are beneficial to increase and standardize the size of oat grains, a condition that allows producers to reach the characters required or desired by the industry.

The analysis of the contribution and relationships of the components of oat grain industrial productivity with nitrogen stimulation yield can generate information seeking more efficient management that benefits the commercialization and processing.

The objective of this study is to measure and interpret the contribution and relationship dynamics of the components of oat industrial quality with grain and industry yield by nitrogen stimulation, partitioning the correlation values in direct and indirect effects by path diagnosis. A proposal that 
jointly aggregates productivity indicators with industrial quality, indicating strategies that promote benefits to the food industry.

\section{Results and Discussion}

\section{Classification of agricultural year}

Table 1 presents information on rainfall, air temperature, and oat grain yield in the soybean/oat and corn/oat cropping systems from 2011 to 2016. 2011 showed welldistributed rainfall during the growing season, especially in the moments before nitrogen application, providing adequate soil moisture for urea solubilization (Figure 1A). Maximum, minimum and average air temperatures remained stable throughout the cycle. In 2013, there was a uniform distribution of rainfall between the months of growing, with accumulated values below the historical average. The timing of $\mathrm{N}$-fertilizer fertilization was also marked by adequate soil moisture due to rainfall in previous days, favoring the use of nutrient by the plant. This year, air temperatures were milder, reducing possible nitrogen losses by volatilization (Figure 1C). Yields above $3 \mathrm{t} \mathrm{ha}^{-1}$ of grains were observed in both fast $\mathrm{N}$-residual (soybean/oat) and slow N-residual (corn/oat) systems. These conditions categorize the years 2011 and 2013 as favorable (FY) to oat grain yield.

In 2012 (Table 1), reduced rainfall was observed at the time of nitrogen application, together with high temperatures, a fact that possibly caused the volatilization of the nutrient. At the end of the cycle, the occurrence of high volume rainfall made it difficult to harvest. In 2014, the first days of the cycle were marked by a significant volume of rainfall above the historical average, along with high temperatures, a condition that can affect photosynthesis efficiency and consequent shoot formation and root growth. At the time of fertilization, although the soil presented satisfactory humidity, the air temperature was high, a condition that may have contributed to nitrogen volatilization, reducing nutrient absorption. These conditions justify the classification of the years 2012 and 2014 as unfavorable (UY) to oat grain yield.

In 2015 (Table 1), accumulated rainfall showed a volume close to the observed average of 25 years. The air temperature and soil moisture conditions were adequate for nitrogen absorption, but a drought period was observed after fertilization, which may have affected crop development (Figure 1E). High temperatures occurred during anthesis, at which time the development of the reproductive system is particularly sensitive to thermal and water stress. In 2016, reduced rainfall was observed during the grain filling period, with heavy rainfall occurring in the final phase of the cycle, which may have negative effects on grain quality. Under these agricultural conditions, the low $\mathrm{C} / \mathrm{N}$ (soybean/oat) ratio system, possibly due to the higher $\mathrm{N}$-residual intake, favored grain yield higher than $3 \mathrm{t} \mathrm{ha}^{-1}$, a fact not found in the high $\mathrm{C} / \mathrm{N}$ (corn/oat) ratio system. These facts classify the years 2015 and 2016 as intermediates (IY) to oat grain yield.

White oat is a highly adaptable crop, but the pursuit of high productivity and quality requires favorable environmental conditions for development (Castro et al., 2012). According to research, a favorable environment requires welldistributed rainfall throughout the growing cycle and in small volumes, with low temperatures from germination to grain filling and higher daytime temperatures in the ripening period (Leonard and Martinelli, 2005). Meteorological factors have a significant effect on the expression of oat productive potential, being air temperature and rainfall the most affecting grain yield and quality (Klink et al., 2014; Silva et al., 2016). Peltonen-Sainioet et al. (2011) showed that high rainfall at the end of the crop cycle has a negative impact on oat productivity. In addition, it can affect quality by reducing hectolitic mass and giving dark color to the grain, an undesirable condition for flake or flour manufacturing industry (Castro et al., 2012). Therefore, upto-date knowledge of meteorological information with management practices is critical for agricultural crops to ensure profitable and sustainable activity (Aseeva and Melnichuk, 2018).

\section{Nitrogen contribution to oat productivity and quality indicators}

In Table 2, the average and relative contribution values in the soybean/oat system indicate that all the analyzed variables present significant contribution of alteration by the effect of nitrogen, except for hectoliter mass. Among these variables, the greatest sensitivity of modification was in the caryopsis mass, showing a tendency of reduction due to the greater use of nitrogen. Grain mass also shows relevant contribution with reduction by nitrogen increase, although in the husking index composition the decreasing tendency is nullified. The caryopsis mass and grain mass are variables directly related to the industrial productivity of oat, indicating that the increase of nutrient to grain yield needs more adjusted criteria. It is noteworthy that the mass of one thousand grains also shows relevant contribution of decrease by the increase of nitrogen dose, and the number of grains greater than $2 \mathrm{~mm}$ shows elevation to the point of $60 \mathrm{~kg} \mathrm{ha}^{-1}$. On the other hand, at the highest dose, the number of grains greater than $2 \mathrm{~mm}$ indicates a reduction trend. In the corn/oat system analysis, the hectoliter mass also showed no significant modification by the use of nitrogen. The greatest magnitude of sensitivity for nitrogen was obtained by the one thousand grain mass, leading to a tendency to decrease its expression. The caryopsis mass and grain mass follow with significant sensitivity due to the increase in nitrogen supply, with decrease in the expression of these indicators. In this system, also the higher nitrogen dose promoted reduction in the number of grains larger than $2 \mathrm{~mm}$. The results presented in the cropping systems by the combined effect of different agricultural years, support the idea that higher nitrogen doses, although increasing grain yield, can cause serious damage to oat grain quality and industrial yield components.

\section{Correlation and trail of oat grain industrial quality}

Table 3 shows the correlation and path analysis of oat grain industrial quality indicators on grain and industry productivity in the use of nitrogen in soybean/oat system. Grain yield shows a positive correlation with the one thousand grain mass, with direct effect via grain yield in the absence and reduced nitrogen dose. On the other hand, increasing nitrogen rates (60 and $120 \mathrm{~kg} \mathrm{ha}^{-1}$ ) promote positive indirect contribution via caryopsis mass and negative indirect contribution through number of grains larger than $2 \mathrm{~mm}$. In the analysis between grain yield and husking index, the negative correlation is observed under the conditions of nitrogen use, indicating that the increase in caryopsis does not guarantee increase in grain yield, but in the total grain mass. The complexity of this relationship becomes evident, mainly due to the different magnitude of 
Table 1. Average values of temperature and precipitation in the months of growing and average grain yield of oats in soybean/oat and corn/oat succession system.

\begin{tabular}{|c|c|c|c|c|c|c|c|c|c|}
\hline \multirow[b]{2}{*}{ Year } & \multirow[b]{2}{*}{ Month } & \multicolumn{3}{|c|}{ Temperature $\left({ }^{\circ} \mathrm{C}\right)$} & \multicolumn{2}{|l|}{ Precipitation $(\mathrm{mm})$} & \multirow{2}{*}{$\begin{array}{c}\mathrm{GY}_{\bar{x} S} \\
\left(\mathrm{~kg} \mathrm{ha}^{-1}\right)\end{array}$} & \multirow{2}{*}{$\begin{array}{c}\mathrm{GY}_{\bar{x} M} \\
\left(\mathrm{~kg} \mathrm{ha}^{-1}\right)\end{array}$} & \multirow[b]{2}{*}{ Class } \\
\hline & & Min & Max & $A v$ & 25 year average* & Occurred & & & \\
\hline \multirow{6}{*}{2011} & June & 7.9 & 18.4 & 13.1 & 136 & 191 & \multirow{6}{*}{$3686 a$} & \multirow{6}{*}{$3122 \mathrm{a}$} & \multirow{6}{*}{ FY } \\
\hline & July & 8.3 & 19.2 & 13.7 & 134 & 201 & & & \\
\hline & August & 9.3 & 20.4 & 14.8 & 122 & 234 & & & \\
\hline & September & 9.5 & 23.7 & 16.6 & 165 & 46 & & & \\
\hline & October & 12.2 & 25.0 & 18.6 & 236 & 211 & & & \\
\hline & Total & - & - & - & 793 & 983 & & & \\
\hline \multirow{6}{*}{2012} & June & 8.8 & 22.0 & 15.4 & 136 & 57 & \multirow{6}{*}{$2378 \mathrm{c}$} & \multirow{6}{*}{1984 c } & \multirow{6}{*}{ UY } \\
\hline & July & 6.4 & 19.7 & 13.0 & 134 & 180 & & & \\
\hline & August & 12.9 & 23.4 & 18.1 & 122 & 61 & & & \\
\hline & September & 12.0 & 23.0 & 17.5 & 165 & 195 & & & \\
\hline & October & 15.0 & 25.5 & 20.2 & 236 & 287 & & & \\
\hline & Total & - & - & - & 793 & 780 & & & \\
\hline \multirow{6}{*}{2013} & June & 8.9 & 20.0 & 14.5 & 136 & 74 & \multirow{6}{*}{3731 a } & \multirow{6}{*}{3269 a } & \multirow{6}{*}{ FY } \\
\hline & July & 7.0 & 20.6 & 13.8 & 134 & 103 & & & \\
\hline & August & 6.6 & 19.8 & 13.2 & 122 & 169 & & & \\
\hline & September & 9.6 & 21.0 & 15.3 & 165 & 123 & & & \\
\hline & October & 13.2 & 27.1 & 20.2 & 236 & 144 & & & \\
\hline & Total & - & - & - & 793 & 613 & & & \\
\hline \multirow{6}{*}{2014} & June & 9.2 & 20.7 & 16.1 & 136 & 412 & \multirow{6}{*}{$2181 d$} & \multirow{6}{*}{$1765 d$} & \multirow{6}{*}{ UY } \\
\hline & July & 9.7 & 21.8 & 15.7 & 134 & 144 & & & \\
\hline & August & 8.8 & 23.7 & 16.2 & 122 & 78 & & & \\
\hline & September & 13.3 & 23.5 & 18.4 & 165 & 275 & & & \\
\hline & October & 16.0 & 27.7 & 21.8 & 236 & 231 & & & \\
\hline & Total & - & - & - & 793 & 1140 & & & \\
\hline \multirow{6}{*}{2015} & June & 9.7 & 21.1 & 15.4 & 136 & 228 & \multirow{6}{*}{$3451 \mathrm{~b}$} & & \\
\hline & July & 10.2 & 18.7 & 14.4 & 134 & 212 & & & \\
\hline & August & 13.4 & 24.6 & 19.0 & 122 & 87 & & $2732 \mathrm{~h}$ & IV \\
\hline & September & 12.4 & 19.6 & 16.0 & 165 & 127 & & $213 \angle D$ & IY \\
\hline & October & 16.1 & 24.8 & 20.4 & 236 & 162 & & & \\
\hline & Total & - & - & - & 793 & 816 & & & \\
\hline & June & 4.7 & 19.3 & 12.0 & 136 & 12 & & & \\
\hline & July & 8.2 & 21.2 & 14.7 & 134 & 81 & & & \\
\hline 2016 & August & 9.4 & 22.5 & 15.9 & 122 & 169 & $3335 \mathrm{~h}$ & $2782 \mathrm{~b}$ & UY \\
\hline & September & 8.4 & 23.8 & 16.1 & 165 & 56 & & & \\
\hline & October & 13.2 & 26.8 & 20.0 & 236 & 326 & & & \\
\hline & Total & - & - & - & 793 & 644 & & & \\
\hline
\end{tabular}

Min = minimum; Max = maximum; $\mathrm{Av}=$ average; $\mathrm{GY}_{\tilde{x} s}=$ average grain yield of soybean/oat system; $\mathrm{GY}_{\bar{x} M}=$ average grain yield of the corn/oat system; ${ }^{*}=\mathrm{Average}$ rainfall obtained from May to October from 1989 to 2016; Averages followed by the same letter in the column do not differ in the probability of $5 \%$ error by the Scott \& Knott test; IY = intermediate year; FY = favorable year; UY = unfavorable year.

Table 2. Average values and relative contribution of nitrogen on industrial quality indicators of oat grains in cropping systems

\begin{tabular}{|c|c|c|c|c|c|c|}
\hline \multirow{2}{*}{ Variables } & \multicolumn{2}{|c|}{$\mathrm{N}$ dose $\left(\mathrm{kg} \mathrm{ha}^{-1}\right)$} & & & \multicolumn{2}{|c|}{ Relative contribution } \\
\hline & 0 & 30 & 60 & 120 & S.j & S.j (\%) \\
\hline & \multicolumn{6}{|c|}{$(2011+2012+2013+2014+2015+2016)$} \\
\hline & \multicolumn{6}{|c|}{ Soybean/oat system } \\
\hline IY & 989 & 1297 & 1492 & 1424 & - & - \\
\hline TGM & 32.39 & 33.24 & 26.55 & 26.33 & 37.88 & 16.65 \\
\hline GM & 1.79 & 1.72 & 1.67 & 1.66 & 42.11 & 20.40 \\
\hline $\mathrm{CM}$ & 1.32 & 1.27 & 1.12 & 1.11 & 88.68 & 36.63 \\
\hline \multirow[t]{2}{*}{$\mathrm{HI}$} & 0.71 & 0.70 & 0.72 & 0.74 & 27.67 & 7.43 \\
\hline & \multicolumn{6}{|c|}{ Corn/oat system } \\
\hline GY & 1628 & 2462 & 3017 & 3328 & - & - \\
\hline$N G_{>2}$ & 55 & 59 & 62 & 57 & 31.71 & 20.34 \\
\hline GM & 1.78 & 1.72 & 1.66 & 1.62 & 12.68 & 10.93 \\
\hline $\mathrm{CM}$ & 1.26 & 1.21 & 1.15 & 1.10 & 10.89 & 21.39 \\
\hline $\mathrm{HI}$ & 0.68 & 0.69 & 0.71 & 0.70 & 4.88 & 4.21 \\
\hline
\end{tabular}




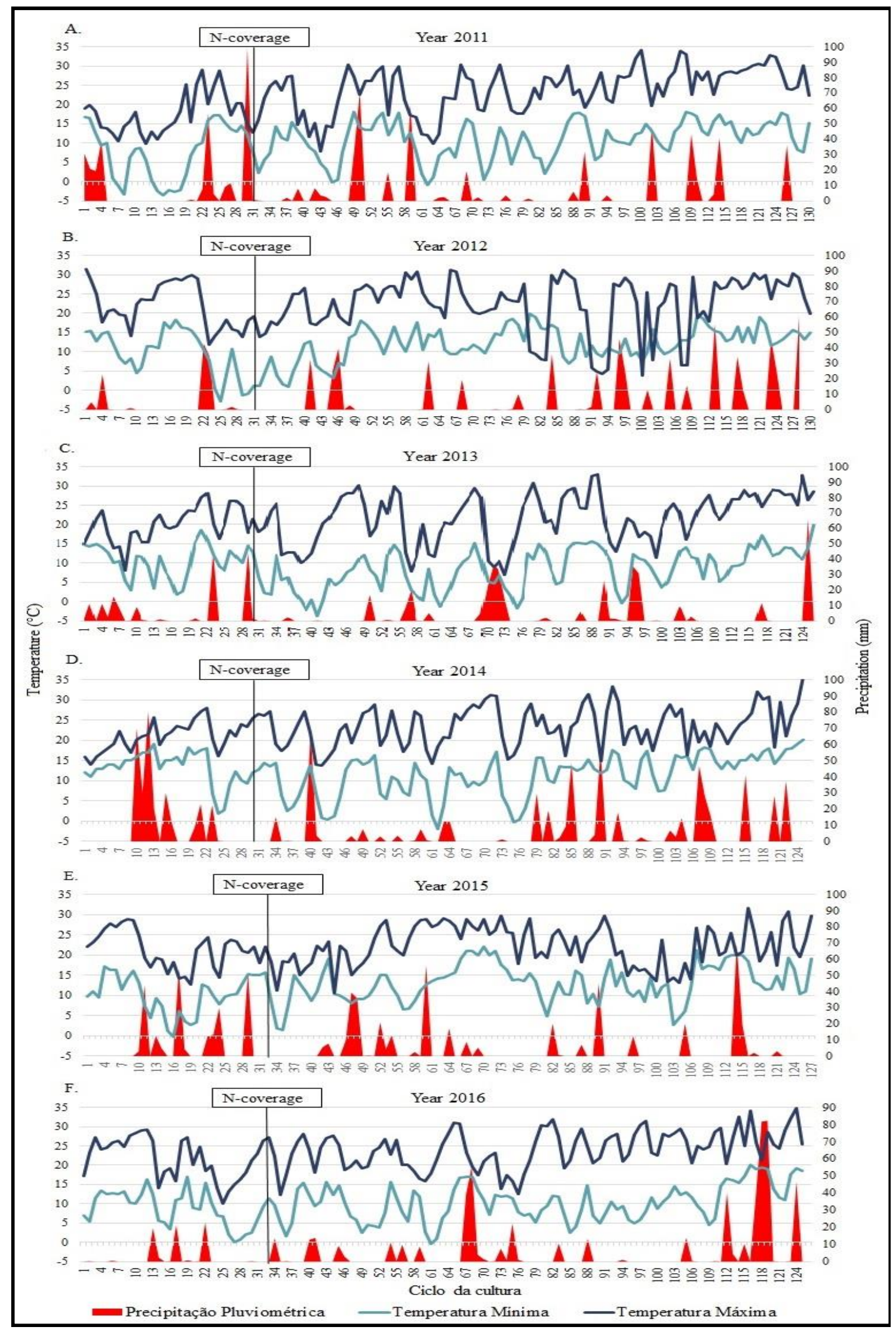

Figure 1. Temperature and precipitation weather data for growing years. 
Table 3. Correlation and path of industrial grain quality indicators on oat grain and industry yield in nitrogen use in soybean/oat system.

\begin{tabular}{|c|c|c|c|c|c|c|c|c|c|c|}
\hline \multirow{2}{*}{ VAR } & \multirow{2}{*}{$\mathrm{EF}$} & \multicolumn{4}{|c|}{$\mathrm{N}$ doses $\left(\mathrm{kg} \mathrm{ha}^{-1}\right)$} & \multirow{2}{*}{$\mathrm{EF}$} & \multicolumn{4}{|c|}{$\mathrm{N}$ doses $\left(\mathrm{kg} \mathrm{ha}^{-1}\right)$} \\
\hline & & 0 & 30 & 60 & 120 & & 0 & 30 & 60 & 120 \\
\hline & \multicolumn{10}{|c|}{$(2011+2012+2013+2014+2015+2016)$} \\
\hline \multirow{7}{*}{ TGM } & $r(G Y x T G M)$ & $0.73^{*}$ & $0.53^{*}$ & $0.50^{*}$ & $0.51^{*}$ & $r(I Y X T G M)$ & 0.27 & $0.45^{*}$ & $0.53^{*}$ & $0.63^{*}$ \\
\hline & D: GY & 0.99 & 0.99 & 0.35 & 0.39 & D: IY & 0.20 & 0.25 & -0.44 & 0.48 \\
\hline & I: HM & 0.09 & -0.13 & 0.22 & -0.12 & $\mathrm{I}: \mathrm{HM}$ & -0.02 & 0.68 & -0.15 & 0.22 \\
\hline & $\mathrm{I}: \mathrm{NG}_{>2}$ & -0.10 & -0.07 & -0.54 & -0.45 & $\mathrm{I}: \mathrm{NG}_{>2}$ & -0.15 & -0.40 & 0.65 & 0.57 \\
\hline & I: GM & 0.05 & -0.15 & -0.05 & 0.03 & I: GM & 0.06 & -0.25 & -0.25 & -0.15 \\
\hline & I: CM & -0.00 & -0.15 & 0.33 & 0.65 & I: CM & 0.11 & 0.15 & 0.38 & 0.52 \\
\hline & $\mathrm{I}: \mathrm{HI}$ & -0.30 & 0.04 & 0.19 & 0.01 & $\mathrm{I}: \mathrm{HI}$ & 0.05 & 0.00 & 0.34 & 0.01 \\
\hline \multirow{7}{*}{$\mathrm{HM}$} & $r(G Y x H M)$ & -0.14 & 0.13 & 0.25 & 0.14 & $r(I Y x H M)$ & $0.59^{*}$ & $-0.32 *$ & $-0.79 *$ & $-0.81^{*}$ \\
\hline & D: GY & 0.77 & 0.37 & 0.49 & 0.27 & D: IY & -0.40 & -0.95 & -0.28 & -0.48 \\
\hline & I: TGM & 0.05 & -0.99 & 0.05 & -0.18 & I: TGM & 0.08 & -0.18 & -0.04 & -0.38 \\
\hline & $\mathrm{I}: \mathrm{NG}_{>2}$ & -0.93 & 0.19 & 0.43 & 0.69 & $\mathrm{I}: \mathrm{NG}_{>2}$ & -0.85 & 0.53 & -0.20 & -0.82 \\
\hline & $\mathrm{I}: \mathrm{GM}$ & 0.39 & 0.39 & 0.03 & -0.04 & I: GM & 0.99 & 0.32 & 0.03 & 0.34 \\
\hline & I: CM & -0.01 & 0.12 & -0.28 & -0.87 & I: CM & 0.70 & -0.07 & -0.10 & 0.38 \\
\hline & $\mathrm{I}: \mathrm{HI}$ & -0.41 & 0.05 & -0.47 & 0.27 & $\mathrm{I}: \mathrm{HI}$ & 0.07 & 0.10 & -0.20 & 0.15 \\
\hline \multirow{7}{*}{$N G_{>2}$} & $r\left(G Y x N G_{>2}\right)$ & 0.17 & -0.08 & -0.19 & -0.11 & $r\left(I Y x N G_{>2}\right)$ & $-0.57^{*}$ & 0.18 & $0.65^{*}$ & $0.34^{*}$ \\
\hline & $\mathrm{D}: \mathrm{GY}$ & 0.95 & -0.20 & -0.79 & -0.72 & D: IY & 0.85 & -0.54 & 0.36 & 0.90 \\
\hline & I: TGM & -0.18 & 0.99 & 0.12 & 0.25 & I: TGM & -0.03 & 0.18 & -0.09 & 0.30 \\
\hline & I: HM & -0.75 & -0.34 & -0.27 & -0.26 & I: HM & 0.37 & 0.94 & 0.15 & 0.48 \\
\hline & I: GM & -0.38 & -0.36 & -0.06 & 0.01 & I: GM & -0.99 & -0.32 & -0.11 & -0.50 \\
\hline & I: CM & 0.04 & -0.12 & 0.40 & 0.85 & I: CM & -0.60 & 0.07 & 0.14 & -0.66 \\
\hline & $\mathrm{I}: \mathrm{HI}$ & 0.49 & -0.05 & 0.41 & -0.22 & $\mathrm{I}: \mathrm{HI}$ & -0.17 & -0.10 & 0.17 & -0.22 \\
\hline \multirow{7}{*}{ GM } & $r(G Y x G M)$ & -0.05 & 0.22 & 0.25 & 0.19 & $r(I Y x G M)$ & $0.75^{*}$ & -0.12 & 0.13 & 0.12 \\
\hline & D: GY & 0.40 & 0.44 & 0.04 & -0.04 & D: IY & 0.99 & 0.37 & 0.25 & 0.57 \\
\hline & I: TGM & 0.06 & -0.95 & -0.03 & -0.10 & I: TGM & 0.10 & -0.17 & 0.06 & -0.13 \\
\hline & I: HM & 0.74 & 0.32 & 0.00 & 0.26 & I: HM & -0.31 & -0.83 & -0.04 & -0.49 \\
\hline & $\mathrm{I}: \mathrm{NG}_{>2}$ & -0.89 & 0.17 & 0.29 & 0.63 & $\mathrm{I}: \mathrm{NG}_{>2}$ & -0.79 & 0.47 & -0.17 & -0.79 \\
\hline & I: CM & -0.01 & 0.16 & -0.28 & -0.83 & I: CM & 0.64 & -0.10 & -0.11 & 0.65 \\
\hline & $\mathrm{I}: \mathrm{HI}$ & -0.36 & 0.05 & 0.23 & 0.27 & $\mathrm{I}: \mathrm{HI}$ & 0.12 & 0.11 & 0.13 & 0.27 \\
\hline \multirow{7}{*}{$\mathrm{CM}$} & $r(\mathrm{GYXCM})$ & -0.05 & -0.16 & -0.09 & -0.36 & $r(I Y x C M)$ & $0.71^{*}$ & $-0.52^{*}$ & $-0.61^{*}$ & -0.03 \\
\hline & D: GY & -0.01 & 0.40 & -0.50 & -0.99 & D: IY & 0.68 & -0.24 & -0.17 & 0.79 \\
\hline & I: TGM & 0.17 & -0.87 & -0.09 & -0.23 & I: TGM & 0.03 & -0.15 & 0.06 & -0.31 \\
\hline & I: HM & 0.75 & 0.11 & 0.28 & 0.19 & I: HM & -0.33 & -0.29 & -0.16 & -0.43 \\
\hline & $\mathrm{I}: \mathrm{NG}_{>2}$ & -0.93 & 0.06 & 0.63 & 0.56 & $\mathrm{I}: \mathrm{NG}_{>2}$ & -0.83 & 0.16 & -0.29 & -0.75 \\
\hline & I:GM & 0.40 & 0.18 & 0.07 & -0.04 & I:GM & 0.99 & 0.15 & 0.15 & 0.47 \\
\hline & $\mathrm{I}: \mathrm{HI}$ & -0.44 & -0.06 & -0.46 & 0.15 & $\mathrm{I}: \mathrm{HI}$ & 0.17 & -0.13 & -0.20 & 0.16 \\
\hline \multirow{9}{*}{$\mathrm{HI}$} & $r(G Y x H I)$ & 0.04 & $-0.40 *$ & $-0.60 *$ & $-0.49 *$ & $r(I Y x H I)$ & $0.41^{*}$ & $-0.37^{*}$ & $-0.87^{*}$ & -0.19 \\
\hline & D: GY & -0.60 & -0.11 & -0.88 & -0.29 & D: IY & 0.09 & -0.22 & -0.37 & -0.30 \\
\hline & I: TGM & 0.64 & -0.02 & -0.02 & -0.01 & I: TGM & 0.11 & 0.00 & 0.01 & -0.01 \\
\hline & I: HM & 0.55 & -0.17 & 0.26 & -0.23 & I: HM & -0.31 & 0.45 & -0.15 & 0.43 \\
\hline & $\mathrm{I}: \mathrm{NG}_{>2}$ & -0.77 & -0.09 & 0.37 & -0.53 & $\mathrm{I}: \mathrm{NG}_{>2}$ & -0.78 & -0.26 & -0.17 & 0.66 \\
\hline & I: GM & 0.24 & -0.22 & -0.04 & 0.04 & I: GM & 0.77 & -0.18 & -0.08 & -0.53 \\
\hline & I: CM & -0.01 & 0.24 & -0.27 & 0.55 & I: CM & 0.53 & -0.14 & -0.09 & -0.43 \\
\hline & Value of $k$ & $2.6 \mathrm{e}^{-2}$ & $4.1 e^{-2}$ & $2.6 \mathrm{e}^{-2}$ & $5.1 e^{-2}$ & Valor k & $2.8 \mathrm{e}^{-2}$ & 0.1 & $4.9 e^{-2}$ & $4.5 e^{-2}$ \\
\hline & $\mathrm{R}^{2}$ & 0.84 & 0.87 & 0.92 & 0.82 & $R^{2}$ & 0.84 & 0.81 & 0.91 & 0.86 \\
\hline
\end{tabular}


Table 4. Correlation and path of industrial grain quality indicators on grain and industry yield in nitrogen use in corn/oat system

\begin{tabular}{|c|c|c|c|c|c|c|c|c|c|c|}
\hline \multirow{2}{*}{ VAR } & \multirow{2}{*}{$\mathrm{EF}$} & \multicolumn{4}{|c|}{$\mathrm{N}$ doses $\left(\mathrm{kg} \mathrm{ha}^{-1}\right)$} & \multirow{2}{*}{$\mathrm{EF}$} & \multicolumn{4}{|c|}{$\mathrm{N}$ doses $\left(\mathrm{kg} \mathrm{ha}^{-1}\right)$} \\
\hline & & 0 & 30 & 60 & 120 & & 0 & 30 & 60 & 120 \\
\hline & \multicolumn{10}{|c|}{$(2011+2012+2013+2014+2015+2016)$} \\
\hline \multirow{7}{*}{ TGM } & $r(G Y x T G M)$ & 0.01 & $-0.59 *$ & $-0.84^{*}$ & -0.12 & r (IYXTGM) & 0.20 & -0.17 & -0.05 & -0.15 \\
\hline & D: GY & 0.49 & -0.69 & -0.60 & -0.13 & I: HM & 0.70 & -0.09 & 0.17 & 0.33 \\
\hline & I: HM & 0.22 & -0.02 & 0.01 & -0.73 & $\mathrm{I}: \mathrm{NG}_{2}$ & -0.19 & 0.13 & -0.03 & -0.73 \\
\hline & $\mathrm{I}: \mathrm{NG}_{2}$ & -0.76 & -0.01 & 0.02 & 0.07 & I: GM & -0.53 & 0.25 & 0.10 & -0.49 \\
\hline & I: GM & 0.07 & 0.07 & -0.03 & 0.40 & $\mathrm{I}: \mathrm{CM}$ & 0.10 & -0.29 & -0.08 & 0.41 \\
\hline & $\mathrm{I}: \mathrm{CM}$ & -0.03 & -0.03 & 0.09 & 0.14 & $\mathrm{I}: \mathrm{HI}$ & -0.03 & -0.21 & 0.03 & 0.31 \\
\hline & $\mathrm{I}: \mathrm{HI}$ & 0.00 & 0.12 & -0.30 & 0.13 & I: HM & 0.11 & 0.05 & -0.26 & 0.00 \\
\hline \multirow{7}{*}{$\mathrm{HM}$} & $r(G Y x H M)$ & -0.13 & -0.10 & 0.05 & -0.08 & $r(I Y x H M)$ & $0.57^{*}$ & -0.20 & $-0.87^{*}$ & -0.25 \\
\hline & $\mathrm{D}: \mathrm{GY}$ & -0.59 & -0.03 & 0.27 & -0.95 & D: IY & 0.67 & 0.25 & -0.61 & -0.95 \\
\hline & I: TGM & -0.04 & -0.35 & -0.03 & -0.10 & I: TGM & -0.12 & -0.05 & 0.01 & 0.26 \\
\hline & $\mathrm{I}: \mathrm{NG}_{2}$ & 0.99 & -0.03 & -0.01 & 0.09 & $\mathrm{I}: \mathrm{NG}_{2}$ & 0.99 & 0.69 & -0.08 & -0.64 \\
\hline & I: GM & -0.30 & 0.17 & 0.00 & 0.61 & $\mathrm{I}: \mathrm{GM}$ & -0.60 & -0.67 & -0.01 & 0.62 \\
\hline & $\mathrm{I}: \mathrm{CM}$ & -0.19 & -0.07 & -0.01 & 0.23 & $\mathrm{I}: \mathrm{CM}$ & -0.45 & -0.52 & 0.00 & 0.52 \\
\hline & I: HI & 0.00 & 0.22 & -0.17 & 0.08 & $\mathrm{I}: \mathrm{HI}$ & 0.08 & 0.09 & -0.15 & 0.00 \\
\hline \multirow{7}{*}{$\mathrm{NG}_{2}$} & $r\left(G Y x N G_{2}\right)$ & -0.18 & 0.08 & -0.13 & -0.06 & $r\left(I Y \times N G_{2}\right)$ & $-0.57^{*}$ & $0.30^{*}$ & $0.56^{*}$ & $0.31^{*}$ \\
\hline & D: GY & -0.99 & 0.03 & 0.05 & -0.09 & D: IY & -0.99 & -0.71 & 0.17 & 0.65 \\
\hline & I: TGM & 0.00 & 0.25 & -0.18 & 0.10 & I: TGM & 0.19 & 0.03 & 0.10 & -0.25 \\
\hline & I: HM & 0.50 & 0.03 & -0.06 & 0.93 & I: HM & -0.62 & -0.24 & 0.27 & 0.93 \\
\hline & I: GM & 0.43 & -0.17 & 0.00 & -0.61 & I: GM & 0.55 & 0.69 & 0.00 & -0.61 \\
\hline & $\mathrm{I}: \mathrm{CM}$ & 0.00 & 0.08 & 0.04 & -0.20 & $\mathrm{I}: \mathrm{CM}$ & 0.25 & 0.58 & 0.00 & -0.45 \\
\hline & I: HI & -0.12 & -0.14 & 0.02 & -0.19 & I: HI & 0.05 & -0.06 & 0.01 & 0.00 \\
\hline \multirow{7}{*}{$\mathrm{GM}$} & $r(G Y \times G M)$ & -0.07 & -0.18 & 0.17 & 0.09 & $r(I Y x G M)$ & $0.45^{*}$ & $-0.51^{*}$ & 0.18 & -0.05 \\
\hline & D: GY & -0.32 & 0.18 & 0.01 & 0.65 & $D: I Y$ & -0.62 & -0.72 & 0.12 & 0.65 \\
\hline & I: TGM & 0.04 & -0.27 & 0.20 & -0.08 & I: TGM & -0.10 & -0.03 & -0.11 & 0.21 \\
\hline & I: HM & -0.60 & -0.03 & -0.02 & -0.90 & I: HM & 0.55 & 0.23 & 0.04 & -0.90 \\
\hline & $\mathrm{I}: \mathrm{NG}_{2}$ & 0.99 & -0.02 & -0.04 & 0.09 & $\mathrm{I}: \mathrm{NG}_{2}$ & 0.99 & 0.68 & 0.00 & -0.61 \\
\hline & $\mathrm{I}: \mathrm{CM}$ & -0.18 & -0.09 & -0.09 & 0.26 & $\mathrm{I}: \mathrm{CM}$ & -0.37 & -0.65 & -0.06 & 0.56 \\
\hline & $\mathrm{I}: \mathrm{HI}$ & 0.00 & 0.05 & 0.11 & 0.07 & $\mathrm{I}: \mathrm{HI}$ & 0.00 & 0.02 & 0.18 & 0.00 \\
\hline \multirow{7}{*}{$\mathrm{CM}$} & $r(G Y \times C M)$ & -0.24 & -0.22 & 0.13 & -0.23 & $r(I Y x C M)$ & $0.45^{*}$ & $-0.69^{*}$ & -0.09 & 0.27 \\
\hline & $\mathrm{D}: \mathrm{GY}$ & -0.28 & -0.09 & -0.19 & 0.30 & $D: I Y$ & -0.52 & -0.69 & -0.07 & 0.69 \\
\hline & I: TGM & 0.03 & -0.21 & 0.28 & -0.08 & I: TGM & 0.04 & -0.03 & -0.08 & 0.15 \\
\hline & I: HM & -0.72 & -0.01 & 0.02 & -0.72 & I: HM & 0.58 & 0.19 & -0.04 & -0.72 \\
\hline & $\mathrm{I}: \mathrm{NG}_{2}$ & 0.96 & 0.00 & 0.00 & 0.07 & I: $N_{2}$ & 0.85 & 0.60 & 0.00 & -0.43 \\
\hline & $\mathrm{I}: G M$ & -0.23 & 0.17 & 0.03 & 0.52 & I:GM & -0.62 & -0.68 & 0.11 & 0.53 \\
\hline & $\mathrm{I}: \mathrm{HI}$ & 0.00 & -0.08 & -0.01 & -0.36 & $\mathrm{I}: \mathrm{HI}$ & 0.14 & -0.05 & -0.01 & 0.01 \\
\hline \multirow{9}{*}{$\mathrm{HI}$} & $r(G Y x H I)$ & $-0.54^{*}$ & $-0.67^{*}$ & $-0.83^{*}$ & $-0.53^{*}$ & $r(I Y x H I)$ & 0.09 & $-0.59 *$ & $-0.64^{*}$ & $0.53^{*}$ \\
\hline & $\mathrm{D}: \mathrm{GY}$ & 0.00 & -0.71 & -0.54 & -0.70 & D: IY & 0.31 & -0.21 & -0.47 & 0.01 \\
\hline & I: TGM & 0.18 & 0.24 & -0.33 & 0.02 & I: TGM & 0.25 & 0.02 & 0.09 & -0.06 \\
\hline & I: HM & -0.05 & 0.00 & 0.08 & 0.11 & I: HM & 0.04 & -0.11 & -0.19 & 0.11 \\
\hline & $\mathrm{I}: \mathrm{NG}_{2}$ & -0.50 & 0.00 & 0.00 & -0.02 & I: $N G_{2}$ & -0.35 & -0.20 & 0.00 & 0.18 \\
\hline & I:GM & 0.04 & -0.09 & -0.02 & -0.06 & I:GM & 0.06 & 0.08 & -0.05 & -0.06 \\
\hline & $\mathrm{I}: \mathrm{CM}$ & -0.20 & -0.11 & 0.00 & 0.15 & I: CM & -0.23 & -0.16 & 0.00 & 0.35 \\
\hline & Value of $k$ & $3.2 \mathrm{e}^{-2}$ & $5.1 e^{-2}$ & $5.1 \mathrm{e}^{-2}$ & $4.9 \mathrm{e}^{-2}$ & Valor k & $4.7 e^{-2}$ & $5.1 \mathrm{e}^{-2}$ & $6.2 \mathrm{e}^{-2}$ & $5.8 \mathrm{e}^{-2}$ \\
\hline & $\mathrm{R}^{2}$ & 0.84 & 0.81 & 0.95 & 0.82 & $\mathrm{R}^{2}$ & 0.82 & 0.85 & 0.95 & 0.80 \\
\hline
\end{tabular}

$\mathrm{VAR}=$ variable; $\mathrm{EF}=$ effect; $\mathrm{GY}=$ grain yield $\left(\mathrm{kg} \mathrm{ha}^{-1}\right) ; \mathrm{IY}=$ industrial yield $\left(\mathrm{kg} \mathrm{ha}^{-1}\right) ; \mathrm{TGM}=$ thousand grain mass $(\mathrm{g}) ; \mathrm{HM}=$ hectolitre mass; $\mathrm{NG}_{>2}=$ number of grains greater than $2 \mathrm{~mm} ; \mathrm{GM}=$ grain mass $(\mathrm{g}) ; \mathrm{CM}=$ caryopsis mass $(\mathrm{g}) ; \mathrm{HI}=$ husking index; $r$ = correlation value; $\mathrm{D}=$ direct contribution; $\mathrm{I}=$ indirect contribution; $\mathrm{R}^{2}=$ coefficient of determination; $\mathrm{k}=$ linearization coefficient; ${ }^{*}=$ Significant at $5 \%$ probability of error, respectively, by $\mathrm{F}$ test.

effects, whether positive or negative under nitrogen use conditions. Industrial productivity shows a positive correlation with the one thousand grain mass under different fertilization conditions (Table 3). This correlation has a positive indirect effect via caryopsis mass and an indirect contribution with the number of grains greater than $2 \mathrm{~mm}$, but with a negative effect on a reduced nitrogen dose and a positive effect on higher doses. Therefore, these conditions indicate that higher doses of $\mathrm{N}$-fertilizer promote increases in the one thousand grain mass, increasing the caryopsis mass and grain size, benefiting the industry productivity. Even, the industry yield shows positive correlation with the number of grains greater than $2 \mathrm{~mm}$ with the highest doses of nitrogen, with more effective contribution directly by the industry yield and indirect by the hectoliter mass. Important information, considering the inclusion of these variables in the determination of oat grain industrial yield. Industry yield shows, in the presence of nitrogen, a negative correlation with hectoliter mass, mainly via direct effect of industry yield. Similarly, industry yield also shows a negative relationship with caryopsis mass, mainly via direct effect by industry yield and indirect effect by hectoliter mass and husking index. The relative contribution of hectoliter mass by nitrogen use was not effective, however, this variable has a strong influence on the determination of caryopsis mass and industry yield. The analysis of industrial yield with the husking index shows a negative correlation in the 30 and $60 \mathrm{~kg} \mathrm{ha}^{-1}$ nitrogen rates, either through direct effect of industrial yield and indirect via number of grains greater than $2 \mathrm{~mm}$. Simultaneously in soybean / oat system, grain and industry yield can be increased by direct increase of one thousand grain mass and indirectly via caryopsis mass. 
Table 4 shows the correlation and path of grain and oat industry yield on indicators of industrial grain quality in the use of nitrogen in the corn/oat system. Grain yield was negatively correlated with one thousand grain mass at intermediate nitrogen application rates (30 and $60 \mathrm{~kg} \mathrm{ha}^{-1}$ ), with strong negative direct action by grain yield. Negative correlation was also observed between grain yield and husking index for all nitrogen use conditions, also by the greater contribution of positive direct effect via grain yield in the presence of nitrogen. Industry yield was positively correlated with the number of grains greater than $2 \mathrm{~mm}$ under nitrogen use conditions, with strong positive direct contribution via industry yield and positive indirect via hectoliter mass (Table 4). The condition without nitrogen fertilization showed a positive correlation between industry yield and grain and caryopsis mass, with negative direct effect via industry yield and positive indirect effect via number of grains greater than $2 \mathrm{~mm}$. At the $30 \mathrm{~kg} \mathrm{ha}^{-1}$ dose of the nutrient, a negative correlation was observed between industry yield and grain and caryopsis mass, with negative direct effect via industry yield and positive indirect effect via number of grains greater than $2 \mathrm{~mm}$. This information highlights the importance of the number of grains larger than $2 \mathrm{~mm}$ for industry yield, influencing grain size and mass.

The correlation between the industrial yield and the husking index is negative in the intermediate doses of nitrogen, mainly due to the negative direct effect of the industrial yield. In the highest dose of nitrogen fertilization the correlation is positive with the main indirect effect via caryopsis mass. In this growing condition, grain yield does not show positive relationships with variables related to industrial grain quality. On the other hand, industrial yield benefits from the increase in the number of grains greater than $2 \mathrm{~mm}$ in nutrient supply. In general, the corn/oat succession system shows distinct behavior regarding the relationships between variables in comparison with the soybean/oat system, indicating that the management proposition in the improvement of grain and industry yield characteristics by nitrogen is dependent on succession systems of high and reduced $\mathrm{N}$-residual release.

The productive potential of oat is due to the efficiency of the plant in terms of nitrogen absorption, remobilization and utilization (Zute et al., 2010; Dumlupinar et al., 2011). Research reports that the character one thousand grain mass of white oats has little constant associations with grain and industry yield, or even nonexistent, and when present, is evidenced reduced magnitude (Hawerroth et al., 2013). However, in this research, in a low carbon/nitrogen ratio succession system, a high positive correlation was observed for both grain and industry yield. This condition was also highlighted by Zute et al. (2010). Industrial yield needs management that increases its physicochemical characteristics and increases the one thousand grain mass and the hectoliter mass, indicative of the amount of reserves that the grain has (Wrobel et al., 2016). According to Browne et al. (2003), higher nutrient supply reduces the hectoliter mass of oat grains. It is noteworthy that Hawerroth et al. (2015) did not show associations of hectoliter mass with grain and industry yield, a characteristic widely used in the selection for grain quality by breeding programs. Buerstmayr et al. (2007) report low or nonexistent relationships between hectoliter mass and oat grain yield, which corroborate the results of this research. In addition, showing high negative correlations with industry yield. According to Hawerroth et al. (2013), industrial yield has positive direct effects with the number of grains greater than two millimeters. Hawerroth et al. (2015) also found positive correlations between industry yield and grain index greater than two millimeters, one thousand grain mass, hectoliter mass, and caryopsis mass. These statements, in general, are also in agreement with the results found, which raises the expectation of the possibility of improvement strategies on grain yield and quality, concomitantly providing agronomic advances and of interest to the food industry.

\section{Materials and methods}

\section{Crop area description}

The works were developed in the field, from 2011 to 2016, in the municipality of Augusto Pestana, RS ( $28^{\circ} 26^{\prime}$ ' $30^{\prime \prime}$ S; 54 '00' 58 " W; altitude $290 \mathrm{~m}$ ). The soil of the experimental area was classified as Typical Dystroferric Red Latosol (Oxisol) and the climate of the region, according to Köppen classification, Cfa type, with hot summer without dry season. The installation area of the experiments has been characterized by direct seeding for twenty years, indicating a consolidated system. In the summer period the area is occupied with soybean and corn, reflecting in the two main cultural precedents of winter crops. In the implementation of the test, about ten days before each sowing, a soil analysis was performed, identifying, on average, the following chemical characteristics of the site: $\mathrm{pH}=6.3 ; \mathrm{P}=$ $34.1 \mathrm{mg} \mathrm{dm}^{-3} ; \mathrm{K}=231 \mathrm{mg} \mathrm{dm}^{-3} ; \mathrm{OM}=3 \% ; \mathrm{Al}=0 \mathrm{cmolc} \mathrm{dm}^{-3}$; $\mathrm{Ca}=6.6 \mathrm{cmolc} \mathrm{dm}^{-3}$ and $\mathrm{Mg}=2.9 \mathrm{cmolc} \mathrm{dm}^{-3}$.

\section{Experimental design}

Sowing was carried out between the first and second week of June with a seeder-fertilizer. Each plot consisted of 5 lines of 5 meters in length each, and line spacing of $0.20 \mathrm{~m}$, corresponding to an experimental unit of $5 \mathrm{~m}^{2}$. The population density was 400 viable seeds per square meter. The seeds of the selected genotypes were submitted to the germination and vigor test in the laboratory in order to correct the plant density to compose the desired population. In the experiments, 60 and $50 \mathrm{~kg} \mathrm{ha}^{-1}$ of $\mathrm{P}_{2} \mathrm{O}_{5}$ and $\mathrm{K}_{2} \mathrm{O}$ were applied at sowing based on soil $\mathrm{P}$ and $\mathrm{K}$ contents for grain yield expectation of $3 \mathrm{tha}^{-1}$, respectively, and $10 \mathrm{~kg} \mathrm{ha}^{-1}$ of $\mathrm{N}$ at basis (except for the standard experimental unit), with the remainder for topdressing to cover the proposed doses of $\mathrm{N}$ fertilizer at the indicated expanded fourth leaf phenological stage, using the urea source. During the execution of the study, tebuconazole fungicide (FOLICUR ${ }^{\circ}$ CE) was applied at a dosage of $0.75 \mathrm{~L} \mathrm{ha}^{-1}$. In addition, weed control was carried out with a metsulfuron-methyl herbicide (ALLY) at a dose of $2.4 \mathrm{~g} \mathrm{ha}^{-1}$ and additional weeding whenever necessary.

The experimental design was a complete randomized block with four replications, following a $4 \times 2$ factorial scheme in the variation sources doses of $\mathrm{N}$-fertilizer $(0,30,60$ and 120 $\mathrm{kg} \mathrm{ha}^{-1}$ ) with urea source, and oat cultivars (Barbarasul and Brisasul), respectively, in soybean/oat and corn/oat succession systems.

\section{Data measurement}

The experiments were harvested to estimate grain yield in each cropping system by hand by cutting the three central lines of each plot. After, in the laboratory, the explanatory variables related to the industrial quality of the oat grains were determined: thousand grain mass (TGM, g), by counting 250 grains and weighing in precision scale, and then multiplied by four; hectoliter mass (HM), by the known volume grain mass of $250 \mathrm{~cm}^{3}$ and converted to $\mathrm{kg} \mathrm{hl}^{-1}$; 
number of grains greater than $2 \mathrm{~mm}\left(\mathrm{NG}_{>2}, \mathrm{n}\right)$, obtained from a sample of 100 grains by counting those above the 2 $\mathrm{mm}$ mesh sieve; grain mass greater than $2 \mathrm{~mm}(\mathrm{GM}, \mathrm{g})$, obtained by weighing 50 grains greater than $2 \mathrm{~mm}$; caryopsis mass (CM, g), obtained by manual husking and weighing of 50 grains greater than $2 \mathrm{~mm}$ and; husking index $\left(\mathrm{HI}, \mathrm{g} \mathrm{g}^{-1}\right)$, by the ratio between the caryopsis mass (CM) and the grain mass (GM) of the 50 grain sample. The main variables considered were grain yield ( $\mathrm{GY}, \mathrm{kg} \mathrm{ha}^{-1}$ ) and industry yield (IY, kg ha ${ }^{-1}$ ), obtained by the product of grain yield (GY), percentage of the number of grains greater than $2 \mathrm{~mm}$ $\left(N G_{>2}\right)$ and the husking index $(\mathrm{HI})$. Therefore, given by

$\mathrm{IY}=\mathrm{GY} \times\left(\frac{\mathrm{NG}_{2}}{100}\right) \times \mathrm{HI}$

\section{Statistical analysis}

Relative contribution analysis by the nitrogen effect by the Mahalanobis distance was performed. Relative contribution was evaluated by the Singh method based on $S_{. j}$ statistics. So, there is:

$D_{\mathrm{ii}^{\prime}}^{2}=\delta^{\prime} \psi^{-1} \delta=\sum_{j=1}^{n} \sum_{j^{\prime}=1}^{n} \omega_{\mathrm{jj}} d_{j} d_{\mathrm{j}^{\prime}}$

where:

- $D_{\mathrm{ii}}^{2}$ : Mahalanobis distance between treatments i and $\mathrm{i}^{\prime}$;

- $\psi$ : residual variance and covariance matrix;

- $\delta^{\prime}=\left[\begin{array}{llll}d_{1} & \mathrm{~d}_{2} & \ldots & \mathrm{d}_{n}\end{array}\right]$, with $d_{j}=Y_{\mathrm{ij}}-Y_{\mathrm{i}^{\prime}}$;

- $Y_{\mathrm{ij}}$ : mean of the $\mathrm{n}^{\text {th }}$ dose in relation to the $\mathrm{j}^{\text {th }}$ character;

- $\omega$ : element of $j^{\text {th }}$ line and $j^{\text {th }}$ column of the inverse matrix of residual variance and covariance.

The total of the distances involving all treatment pairs is given by,

$$
\sum_{i<i^{\prime}} \sum_{i i^{\prime}}^{2}=\sum_{m} D_{m}^{2}=\sum_{j=1} S_{. j}
$$

where the percentages of $S_{. j}$ are the measure of the relative importance of the variable $\mathrm{j}$.

The magnitude and direction of correlations between grain yield and industry yield with the industrial quality components of oat grain were determined. The hypotheses were tested at a $5 \%$ error probability level by adopting the ttest, considering $\mathrm{n}-2$ degrees of freedom and following the model $t=r /\left[\sqrt{\left(1-r_{2}\right)} /(n-2)\right]$, where $r$ is the correlation coefficient between the characters $X$ and $Y$ and $n$ $=$ degrees of freedom in the considered treatment levels. In the correlation analysis, the joint effect of the variation sources of agricultural and cultivar years was considered, since according to Krüger et al. (2011), the inclusion of variation sources in the correlation model is an effective way of knowing with greater fidelity the strength of these relationships. Afterwards, path analysis was performed to detect direct and indirect effects of variables on grain and industry yield by the decomposition of phenotypic correlation, which includes simultaneously parts attributed to genetic and environmental effects. Being $Y$ (grain and industry yield) the main variable resulting from the joint action of other variables $X_{n}$ (components of industrial quality), obtained by the model:

$Y=\beta_{1} X_{1}+\beta_{2} X_{2}+\ldots+\beta_{n} X_{n}+\varepsilon$

where $X_{1}, X_{2}, \ldots, X_{n}$ are explanatory variables and $Y$ is the main (or dependent) variable.

Seeing,

$y=\frac{Y-\bar{Y}}{\sigma_{y}}$
$x=\frac{X_{i}-\bar{X}_{i}}{\sigma_{\mathrm{xi}}}$

$u=\frac{\varepsilon}{\hat{\sigma}_{\varepsilon}}$

$p=\frac{\hat{\sigma}_{\varepsilon}}{\hat{\sigma}_{y}}$

$p_{\mathrm{oi}}=\frac{b_{\mathrm{oi}} \hat{\sigma}_{\mathrm{xi}}}{\hat{\sigma}_{y}}$

there is,

$y=p_{1} x_{1}+p_{2} x_{2}+\ldots+p_{n} x_{n}+p_{\varepsilon} u$

Through this model, the direct and indirect effects of the explanatory variables on the main variable were obtained $(1$ The path coefficients were estimated from the system of equations $X^{\prime} X\left\{\hat{\beta}=X^{\prime} Y\right.$,

$\mathbf{X}^{\prime} \mathbf{Y}=\left[\begin{array}{c}r_{1 \mathrm{y}} \\ r_{2 \mathrm{y}} \\ \vdots \\ r_{\mathrm{ny}}\end{array}\right]$

$\mathbf{X}^{\prime} \mathbf{X}=\left[\begin{array}{cccc}1 & r_{12} & \cdots & r_{1 \mathrm{n}} \\ r_{12} & 1 & \cdots & r_{2 \mathrm{n}} \\ \cdots & \cdots & \cdots & \cdots \\ r_{1 \mathrm{n}} & r_{2 \mathrm{n}} & \cdots & 1\end{array}\right]$

$\beta=\left[\begin{array}{c}p_{1} \\ p_{2} \\ \vdots \\ p_{n}\end{array}\right]$

Thus,

$r_{\mathrm{ij}}=p_{i}+\sum_{j \neq 1}^{n} p_{j} r_{\mathrm{ij}}$

where:

- $r_{\mathrm{ij}}$ : correlation between the main variable $(\mathrm{y})$ and the $\mathrm{i}^{\text {th }}$ explanatory variable;

- $p_{i}$ : measure of the direct effect of variable $i$ on the main variable;

- $\quad p_{j} r_{\mathrm{ij}}$ : measure of indirect effect of variable $\mathrm{i}$, via variable $\mathrm{j}$, on the main variable.

The coefficient of determination of the path diagram is given by:

$\mathrm{R}^{2}=p_{2} r_{1 \mathrm{y}}+p_{2} r_{2 \mathrm{y}}+\ldots+p_{n} r_{\mathrm{ny}}$

and the residual effect is estimated by:

$p_{\varepsilon}=\sqrt{1-\mathrm{R}^{2}}$

The analyzes of relative contribution and correlation and path were performed using the computer program GENES (Quantitative Genetics and Experimental Statistics, version 2015.5.0).

\section{Conclusions}

The increase of nitrogen promotes greater change in the caryopsis mass in soybean/oat system and the one thousand grain mass and number of grains greater than $2 \mathrm{~mm}$ in corn/oat system, with a tendency of reduction.

In soybean/oat system, grain and industry yield can be simultaneously increased by direct increase via one thousand grain mass and indirect increase by caryopsis mass. In corn/oat system the grain yield does not show any relationship with industrial quality variables, however, the industry yield is benefited by the ip\&rease of the number of grains larger than $2 \mathrm{~mm}$. The management proposition in the improvement of the grain and industry yield characteristics by nitrogen is dependent on the succession systems of high and reduced $\mathrm{N}$-residual release systems.

(5) 


\section{Acknowledgments}

The authors would like to thank the Graduate Program in Mathematical Modeling at UNIJUI for the resources available in the development of this research, which was part of the doctoral thesis of the first author of this work and CNPq, FAPERGS, CAPES for the scholarships, Scientific and Technological Initiation and Research Productivity.

\section{References}

Arenhardt EG, Silva JAG, Gewehr E, Oliveira AC, Binello MO, Valdiero AC, Gzergorczick ME, Lima ARC (2015) The supply of nitrogen in wheat yield by year condition and succession system in southern Brazil. African Journal of Agricultural Research. 10: 4322-4330.

Aseeva TA, Melnichuk IB (2018) Dependence of various oat ecotypes' yield capacity on climatic factors in the middle Amur region. Russian Agricultural Sciences. 44: 5-8.

Browne RA, Branco EM, Burke JI (2003) Effect of nitrogen, seed rate and plant growth regulator (chlormequat chloride) on the grain quality of oats (Avena sativa). The Journal of Agricultural Science. 141: 249-258.

Buerstmayr H, Krenn N, Stephanb U, Grausgruberc H, Zechner E (2007) Agronomic performance and quality of oat (Avena sativa L.) genotypes of worldwide origin produced under Central European growing conditions. Field Crops Research. 101: 343-351.

Castro GSA, Costa CHM, Neto JF (2012) Ecophysiology of oats. Scientia Agraria Paranaensis 11: 1-15

Dumlupinar Z, Maral H, Kara R, Dokuyucu T, Akkaya A (2011) Evaluation of Turkish oat landraces based on grain yield, yield components and some quality traits. Turk J Field Crops. 16: 190-196.

Hawerroth MC, Carvalho FIF, Oliveira AC, Silva JAG, Gutkoski LC, Sartori JF, Woyann LG, Barbieri RL, Hawerroth FJ (2013) Adaptability and stability of white oat cultivars in relation to chemical composition of the caryopsis. Pesquisa Agropecuária Brasileira. 48: 42-50.

Hawerroth MC, Silva JAG, Woyabb LG, Zimmer CM, Groli EL, Oliveira AC, Carvalho FIF (2015) Correlations among industrial traits in oat cultivars grown in different locations of Brazil. Australian Journal of Crop Science. 9: 1182-1189.

Klink K, Wiersma JJ, Crawford CJ, Stuthman DD (2014) Impacts of temperature and precipitation variability in the Northern Plains of the United States and Canada on the productivity of spring barley and oat. International Journal of Climatology. 34: 2805-2818.

Krüger CAMB, Medeiros SLP, Silva JAG, Gaviraghi J (2011) Heritability and phenotypic correlation of characters related to grain yield and morphology of canola. Pesquisa Agropecuária Brasileira. 46: 1625-1632
Leonard KJ, Martinelli JA (2005) Virulence of Oat Crown Rust in Brazil and Uruguay. Plant Disease. 89: 802-808.

Marolli A, Silva JAG, Romitti MV, Mantai RD, Scremin OB, Frantz RZ, Sawicki S, Arenhardt EG, Gzergorczick ME, Lima ARC (2017) Contributive effect of growth regulator Trinexapac-Ethyl to oats yield in Brazil. African Journal of Agricultural Research. 12: 795-804.

Martinez MF, Arelovich HM, Wehrhahne LN (2010) Grain yield, nutrient content and lipid profile of oat genotypes grown in a semiarid environment. Field Crops Research. 116: 92-100.

Obur A, Holman JD, Schlegel A (2018) Seeding rate and nitrogen application effects on spring oat and triticale forage. Kansas Agricultural Experiment Station Research Reports. 4: 1-8.

Scremin OB, Silva JAG, De Mamann ATW, Marolli A, Mantai $R D$, Trautmann APB, Kraisig AR, Scremin AH, Krüger CAMB, Dornelles EF (2017) Nitrogen and hydrogel combination in oat grains productivity. International Journal of Development Research. 7: 13896-13903.

Silva JAG, Goi Neto CJ, Fernandes SB, Mantai RD, Scremin OB, Pretto R (2016) Nitrogen efficiency in oats on grain yield with stability. Revista Brasileira de Engenharia Agrícola e Ambiental. 20: 1095-1100.

Silveira SFS, Hawerroth MC, Oliveira DCS, Woyann LG, Almeida HCF, Luz VK, Thurow L, Silva RM, Gutkoski LC, Maia LC, Oliveira AC (2016) Heterosis and genetic parameters for grain quality in oat segregating populations. Scientia Agricola. 73: 471-477.

Takayama T, Ishikawa N, Taya S (2006) The effect to the protein concentration and flour quality of nitrogen fertilization at 10 days after heading in wheat. Japan Agricultural Research Quarterly. 40: 291-297.

Tomple BM, Hwan JI (2018) Enhancing seed productivity and feed value of oats (Avena Sativa L.) with different seeding rate and nitrogen fertilizing levels in Gyeongbuk area Byamungu Mayange. Journal of Agriculture \& Life Science. 52: 61-72

White E, Finnan J (2017) Crop structure in winter oats and the effect of nitrogen on quality-related characters. Journal of Crop Improvement. 31: 758-779

Wrobel FL, Neumann M, Leão GFM, Horst EH, Ueno RK (2016) Nitrogen doses under productivity and nutritional aspects of dual purpose wheat grains and straw. Revista Acadêmica Ciência Animal. 14: 27-35

Ziech ARD, Conceição PC, Heberle CT, Cassol C, Balim NM (2016) Produtividade e componentes de rendimento de milho em função de plantas de cobertura e doses de nitrogênio. Revista Brasileira de Milho e Sorgo. 15: 195201

Zute S, Vicupe Z, Gruntina M (2010) Factors influencing oat grain yield and quality under growing conditions of West Latvia. Agronomy Research. 8: 749-754. 